Research Paper

\title{
Lower-dose decitabine improves clinical response compared with best supportive care in lower-risk MDS patients: a prospective, multicenter phase 2 study
}

\author{
Li Ye ${ }^{1,2}$, Chen Mei ${ }^{1,2}$, Yanling Ren ${ }^{1,2}$, Xinping Zhou ${ }^{1,2}$, Liya Ma ${ }^{1,2}$, Weilai Xu ${ }^{1,2}$, Juying Wei ${ }^{1,2}$, Huifang Jiang ${ }^{3}$, \\ Liming Zhang ${ }^{4}$, Hui Zeng5, Hongyan Tong1, ${ }^{\boxplus}$ \\ 1. MDS Center, Department of Hematology, the First Affiliated Hospital, Zhejiang University School of Medicine, Hangzhou 310003, Zhejiang Province, China. \\ 2. Key Laboratory of Hematologic Malignancies of Zhejiang Province, Hangzhou 310009, Zhejiang Province, China. \\ 3. Department of Hematology, Tongde Hospital of Zhejiang Province, Hangzhou 310012, Zhejiang Province, China. \\ 4. Department of Hematology, Zhuji People's Hospital of Zhejiang Province, Zhuji 311800, Zhejiang Province, China. \\ 5. Institute of Hematology, the First Hospital of Jiaxing City in Zhejiang Province, Jiaxing 314001, Zhejiang Province, China. \\ $\triangle$ Corresponding author: Hongyan Tong, MDS Center, Department of Hematology, the First Affiliated Hospital, Zhejiang University School of Medicine, \#79 Qingchun \\ Road, Hangzhou, Zhejiang Province, P.R. China, 310003. Tel: 86-571-87235589, Fax: 86-571-87236702. E-mail: tonghongyan@zju.edu.cn.
}

(c) The author(s). This is an open access article distributed under the terms of the Creative Commons Attribution License (https://creativecommons.org/licenses/by/4.0/). See http://ivyspring.com/terms for full terms and conditions.

Received: 2020.11.20; Accepted: 2021.03.04; Published: 2021.03.19

\begin{abstract}
Purpose: To explore the efficacy and safety of lower-dose decitabine in patients with lower-risk MDS, a prospective multicenter phase II study was conducted to compare decitabine with the best supportive care (BSC).

Methods: Patients diagnosed with lower-risk MDS from September 2013 to August 2018 were assigned to the decitabine group or the BSC group. Decitabine $\left(12 \mathrm{mg} / \mathrm{m}^{2} /\right.$ day $)$ was administered over 1 hour/day for 5 consecutive days in a 4-week cycle. BSC, including growth factors, transfusion, thalidomide, lenalidomide, and immunosuppressive agents were given consecutively. The endpoints included the proportion of patients who achieved overall response (OR) in the first 2 or 3 courses, event-free survival (EFS), and overall survival (OS).

Results: A total of recruited 82 patients were analyzed. In the decitabine group, $65.9 \%$ (27/41) achieved OR after 2 or 3 cycles of treatment, compared with $22.0 \%(9 / 41)$ in the BSC group $(p<0.01)$. Besides, $44.0 \%(11 / 25)$ in the decitabine group became independent of RBC/Platelets transfusion, compared with $27.8 \%(5 / 18)$ in the BSC group. Patients with gene mutation and treated with decitabine achieved a higher OR rate, compared with those without gene mutation $[72.0 \%(18 / 25)$ vs $11.5 \%(3 / 26), p<0.01]$. There was no significant difference in the median EFS between the decitabine and BSC groups (20.6 vs 14.3 months respectively, $P=0.665)$. In the decitabine group, the most significant adverse events were infections of any grades or neutropenic fever $(46.3 \%, 19 / 41)$ and one patient $(4.2 \%)$ died of acute cerebral infarction within 6 weeks of treatment.

Conclusion: Lower-dose decitabine demonstrated promising clinical response with acceptable toxicity profiles in patients with low- and intermediate 1-risk MDS. A higher response rate to decitabine was observed in patients with mutated genes. Therefore, lower-dose decitabine can be advocated for patients with low-risk MDS and mutated genes.
\end{abstract}

Key words: myelodysplastic syndromes; lower risk; hypomethylating

\section{Introduction}

Myelodysplastic syndromes (MDS) are characterized by peripheral cytopenias and dysplastic changes in bone marrow [1-3]. Based on the International Prognostic Scoring System (IPSS) and the Revised IPSS (R-IPSS), MDS patients can be stratified according to the risk category (lower- and higher-risk). However, to enable the identification of patients with a lower-risk disease who may benefit from early treatment, Garcia-Manero et al. [4] developed a novel prognostic model (Lower-Risk PSS, 
LR-PSS), whereby patients with lower-risk MDS were further divided into 3 categories and demonstrated that patients in category 2 or 3 were associated with a poor prognosis if untreated. This was then independently validated by Bejar R et al. [5], which showed similar survival outcomes between lower-risk patients in category 3 and those categorized as the intermediate-2 risk in the IPSS. However, these risk-predicting models have not taken the factor of gene mutation into consideration, with several studies consistently associating gene mutation with increased levels of risk and shortened overall survival [6-8]. A subgroup of lower-risk patients with poorer prognosis may benefit from early intervention.

There are several options of treatment for patients with lower-risk MDS, which include growth factors, transfusion, lenalidomide, hypomethylating agents, and immunosuppressive therapy. Decitabine represents a classic hypomethylating agent. To date, the optimal dose of decitabine in treating lower-risk patients has not been well established. Studies have examined decitabine $20 \mathrm{mg} / \mathrm{m}^{2} /$ day for 5 days in lower-risk MDS patients, and revealed an overall response (OR) rate of approximately $55 \%$, with a significantly longer OS observed among those who achieved hematologic improvement (HI) [9, 10]. However, several clinical trials have also shown that a reduced dose of decitabine conferred a comparable response while minimizing treatment-related toxicities [11-15]. The study by Yang AS et al. [12] has revealed that lower doses $\left(5-20 \mathrm{mg} / \mathrm{m}^{2} /\right.$ day $)$ of decitabine for longer periods (5-14 days) were feasible in maintaining an advantage of its demethylating properties, which was consistent with another study [13]. Furthermore, decitabine $20 \mathrm{mg} / \mathrm{m}^{2} /$ day for 3 days has also been shown to have a promising efficacy in lower-risk MDS patients [15]. Reassuringly, the study by Jabbour E et al. [14] demonstrated an improved OR rate in patients treated with low-dose decitabine $(70 \%, 49 / 70)$.

Therefore, we postulated that lower-dose decitabine $\left(12 \mathrm{mg} / \mathrm{m}^{2} /\right.$ day $)$ for consecutive 5 days might be optimal for patients with lower-risk MDS, and a phase 2 trial was conducted to compare low-dose decitabine with a single best supportive care (BSC) treatment.

\section{Methods}

\section{Patients}

Adult patients diagnosed with MDS between September 2013 to August 2018 and categorized as low-risk or intermediate 1-risk of the IPSS based on 2008 WHO classification [16] were eligible for this study. The patient inclusion criteria included an adequate Eastern Cooperative Oncology Group performance status (0-2), normal organ function (bilirubin $<2 \times$ upper normal limit and creatinine $<2 \times$ upper normal limit), and high Epo levels (more than $500 \mathrm{mU} / \mathrm{mL}$ ). Patients were excluded if they met any of the following criteria: (1) secondary MDS; (2) hypocellular marrows; (3) isolated del (5q) with or without one other abnormality except $-7 /$ del (7q); (4) previously received chemotherapy, azacitidine, or decitabine before this study; (5) uncontrolled intercurrent illness; (6) active or uncontrolled infection; (7) estimated to have short survival ( $<3$ months); (8) nursing or pregnant.

This study was approved by the Ethics Committee of the First Affiliated Hospital, Zhejiang University School of Medicine, and was registered with the Chinese Clinical Trial Registry (CHiCTRIPR-15006454). Informed consent was obtained from all patients, conforming to the institutional guidelines and in accordance with the Declaration of Helsinki.

\section{Treatment regimens}

Based on the clinical guidelines and patients' preferred option of the treatment regimen, they were assigned to either the decitabine group or the BSC group. The dosage of decitabine was $12 \mathrm{mg} / \mathrm{m}^{2} /$ day intravenously over 1 hour on day 1 to day 5 . The BSC treatments included administering growth factors, blood transfusion, thalidomide, lenalidomide, and immunosuppressive agents. When the patient failed to achieve any clinical response after 3 cycles of treatment, other treatment regimens would be instituted. On the other hand, responders could choose to undergo allogeneic hematopoietic stem cell transplantation (allo-HSCT), or consolidation courses, such as the previous regimen, or watch and wait. The duration of the consolidation treatment was until disease relapse, the progression of the disease, withdrawal of consent, intercurrent illness, or incidence of severe adverse events in the judgment of the investigators. Each course was administered every 4 weeks, as long as there were no significant myelosuppressive events, life-threatening complications such as severe infection, bleeding, or severe organ damage; otherwise, the interval could be interrupted, or extended up to 6-8 weeks. Blood transfusion, G-CSF, antimicrobial, and antifungal therapy were administered at the discretion of the physicians.

\section{Clinical response and toxicity}

The primary outcome was the OR rate, which was assessed in the first 3 cycles according to the modified International Working Group 2006 criteria [17], including the complete remission (CR), partial response (PR), marrow $\mathrm{CR}(\mathrm{mCR})$, and hematologic 
improvement (HI). The secondary outcomes included overall survival (OS), event-free-survival (EFS), and transfusion independence. The OS was calculated as the number of months from the start of the therapy to the day of death or allo-HSCT, while the EFS represented the number of months from treatment initiation to disease progression, relapse, or death, whichever occurred first. The common terminology criteria (CTCAE v3.0) [18] were used for assessing toxicities.

\section{DNA sequencing}

A target-specific next-generation sequencing (NGS) approach was used, which combined multiplex PCR-based target enrichment and library generation with ultra-deep high-throughput parallel sequencing using an Ion Proton platform $[19,20]$. The target genes were SF3B1, SRSF2, U2AF1, DNMT3A, IDH1, IDH2, TET2, ASXL1, EZH2, RUNX1, ETV6, NRAS, JAK2, $C B L$, and TP53. Mutations were annotated using multiple databases, including 1000 Genomes, COSMIC, PolyPhen-2, and dbSNP.

\section{Statistical analysis}

All statistical analyses were performed using SPSS 22.0 software. A $P$-value of $<0.05$ (2-sided) was considered statistically significant. Categorical variables were analyzed with the $\chi^{2}$ test or Fisher's exact test (for small samples), while continuous variables were analyzed with the Student's t-test. Survival curves were constructed by the KaplanMeier method followed by the log-rank test. Factors associated with OR, EFS, and OS were analyzed using a stepwise backward selection approach by the logistic or COX regression.

\section{Results}

\section{Patient characteristics}

A total of 88 patients across 4 treatment centers in China were enrolled in this study, with 82 patients available for data analyses (Figure 1). Of all patients receiving decitabine, 6 patients received only one cycle of decitabine and the treatment had to be discontinued due to the development of severe toxicity. The median number of treatment cycles in the decitabine group was 3 (IQR: 3-5 cycles). There were 5 patients undergoing allo-HSCT following the treatment with decitabine. In the BSC group, most patients received growth factors combined with thalidomide or lenalidomide $(n=13)$, growth factors $(n=13)$, and immunosuppressive agents $(n=6)$. There were $85.4 \%(70 / 82)$ of patients classified as intermediate 1-risk MDS by IPSS, while $98.8 \%(81 / 82)$ of patients were in category 2 or 3 by LR-PSS. Both study groups demonstrated comparable baseline characteristics (Table 1).

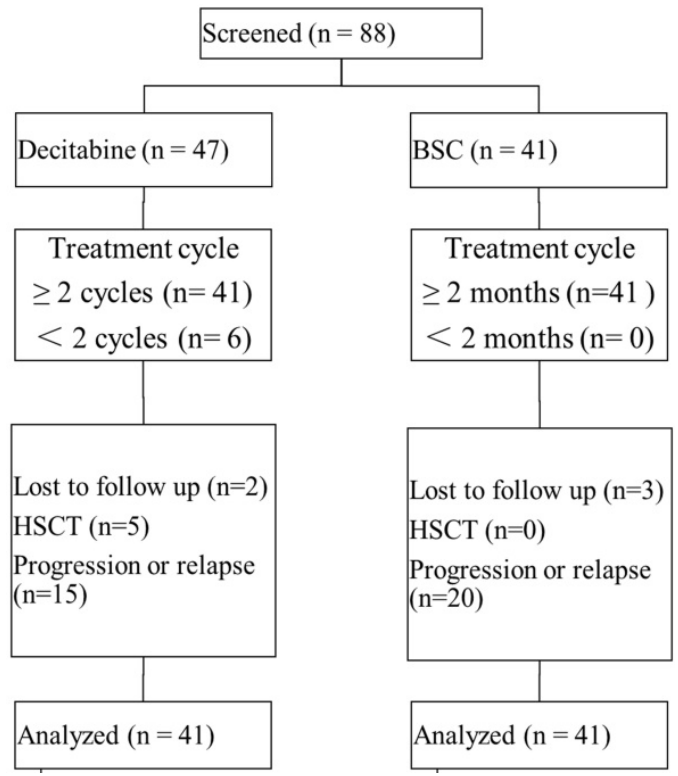

Figure 1. Patient disposition. Abbreviations: BSC: best supportive care; HSCT: hematopoietic stem cell transplantation.

Table 1. Patient characteristics

\begin{tabular}{|c|c|c|c|}
\hline & Decitabine $(n=41)$ & $\operatorname{BSC}(n=41)$ & $p$ value \\
\hline Sex, Male/Female & $25 / 23$ & $43 / 21$ & 0.481 \\
\hline Median age, (IQR; years) & $59(44-68)$ & $61(52-69)$ & 0.299 \\
\hline Neutrophil count, $\left(\mathrm{IQR} ; \times 10^{9} / \mathrm{L}\right)$ & $1.2(0.6-1.7)$ & $1.0(0.7-1.9)$ & $>0.99$ \\
\hline Hemoglobin level, (IQR; g/L) & $84(66-109)$ & $78(59-93)$ & 0.164 \\
\hline Platelet count, $\left(\mathrm{IQR} ; \times 10^{9} / \mathrm{L}\right)$ & $43(19-116)$ & 49 (31-95) & 0.687 \\
\hline BM blasts percentage, (IQR; \%) & $5(2-7)$ & $4(2-5)$ & 0.076 \\
\hline Transfusion dependence, $\mathrm{n}(\%)$ & $25(61.0 \%)$ & $18(43.9 \%)$ & 0.122 \\
\hline WHO classification, $\mathbf{n}(\%)$ & & & 0.085 \\
\hline RCUD & $7(17.1 \%)$ & $3(7.3 \%)$ & \\
\hline RARS & $1(2.4 \%)$ & $2(4.9 \%)$ & \\
\hline RCMD & $13(31.7 \%)$ & $23(56.1 \%)$ & \\
\hline RAEB1 & $20(48.8 \%)$ & $13(31.7 \%)$ & \\
\hline Baseline cytogenetic, n (\%) & & & 0.155 \\
\hline $\operatorname{del}(20 q)$ & $2(4.9 \%)$ & $1(2.4 \%)$ & \\
\hline+8 & $3(7.3 \%)$ & $5(12.2 \%)$ & \\
\hline other & $4(9.8 \%)$ & 0 & \\
\hline Complex ( $\geq$ three abnormalities) & $1(2.4 \%)$ & 0 & \\
\hline IPSS risk, $\mathrm{n}(\%)$ & & & 0.885 \\
\hline Low & $5(12.2 \%)$ & $3(7.3 \%)$ & \\
\hline Intermediate-1 & $34(82.9 \%)$ & $36(87.8 \%)$ & \\
\hline IPSS-R risk, n (\%) & & & 0.258 \\
\hline Low & $8(19.5 \%)$ & $9(22.0 \%)$ & \\
\hline Intermediate & $16(39.0 \%)$ & $22(53.7 \%)$ & \\
\hline High & $14(34.1 \%)$ & $8(19.5 \%)$ & \\
\hline Very high & $1(2.4 \%)$ & 0 & \\
\hline LR-PSS & & & 0.172 \\
\hline Category 1 (score $0-2$ ) & 0 & $1(2.4 \%)$ & \\
\hline Category 2 (score $3-4$ ) & $18(43.9 \%)$ & $24(58.5 \%)$ & \\
\hline Category 3 (score 5-7) & $21(51.2 \%)$ & $14(34.1 \%)$ & \\
\hline
\end{tabular}

NGS was performed in 75 patients (91.5\%) before the commencement of treatment. At least 1 mutation was detected in $41(62.2 \%)$ patients. The most frequently detected mutations were TET2 $(13 / 71$, $18.3 \%)$, U2AF1 (13/77, 16.9\%), SF3B1 (9/77, 11.7\%), 
and SRSF2 (7/77, 9.1\%). The incidence of each gene mutation was also comparable between the two groups (Table 2).

\section{Treatment response}

The rates of OR and $\mathrm{CR}$ for the entire cohort were $43.9 \%$ and $12.2 \%$ respectively. Patients treated with decitabine had significantly higher OR $(65.9 \%$ vs $22.0 \%, \mathrm{p}<0.01)$ and $\mathrm{CR}(19.5 \%$ vs $4.9 \%, \mathrm{p}=0.043)$ rates compared with those treated with BSC (Table 3). The overall transfusion-dependent rate before treatment was $52.4 \%(43 / 82)$, which comprised of 25 patients in the decitabine group and 18 in the BSC group. Following treatment, $44 \%$ of patients in the decitabine group became transfusion-independent compared with $27.8 \%$ in the BSC group $(p=0.278)$.

Table 2. Gene mutation status

\begin{tabular}{llll}
\hline Gene & Decitabine $(\mathrm{n}=41), \mathrm{N}(\%)$ & $\mathrm{BSC}(\mathrm{n}=41), \mathrm{N}(\%)$ & $p$ value \\
\hline SF3B1 & $3 / 39(7.7 \%)$ & $6 / 38(15.8 \%)$ & 0.310 \\
SRSF2 & $5 / 39(12.8 \%)$ & $2 / 38(5.3 \%)$ & 0.431 \\
U2AF1 & $5 / 39(12.8 \%)$ & $8 / 38(21.1 \%)$ & 0.335 \\
DNMT3A & $2 / 39(5.1 \%)$ & $1 / 38(2.6 \%)$ & $>0.99$ \\
IDH1 & $1 / 39(2.6 \%)$ & $1 / 38(2.6 \%)$ & $>0.99$ \\
IDH2 & $3 / 39(7.7 \%)$ & $0 / 37$ & 0.241 \\
TET2 & $6 / 37(16.2 \%)$ & $7 / 34(20.6 \%)$ & 0.634 \\
EZH2 & $1 / 37(2.7 \%)$ & $2 / 34(5.9 \%)$ & 0.604 \\
ASXL1 & $2 / 37(5.4 \%)$ & $4 / 34(11.8 \%)$ & 0.417 \\
RUNX1 & $3 / 37(8.1 \%)$ & $3 / 34(8.8 \%)$ & $>0.99$ \\
ETV6 & $1 / 37(2.7 \%)$ & $0 / 34$ & $>0.99$ \\
JAK2 & $1 / 37(2.7 \%)$ & $2 / 34(5.9 \%)$ & 0.604 \\
TP53 & $2 / 37(5.4 \%)$ & $0 / 34$ & 0.494 \\
NRAS & $0 / 34$ & $1 / 34(2.9 \%)$ & 0.479 \\
CBL & $0 / 37$ & $0 / 34$ & -
\end{tabular}

Table 3. Treatment response

\begin{tabular}{|c|c|c|c|}
\hline & Decitabine $(\mathrm{n}=41)$ & $\operatorname{BSC}(n=41)$ & $p$ value \\
\hline \multicolumn{4}{|c|}{ Response rate, $\mathbf{N}(\%)$} \\
\hline CR & $8(19.5 \%)$ & $2(4.9 \%)$ & $0.043^{*}$ \\
\hline PR & 0 & 0 & - \\
\hline $\mathrm{mCR}$ & $9(22.0 \%)$ & $1(2.4 \%)$ & $0.007^{*}$ \\
\hline $\mathrm{HI}$ & $14(34.1 \%)$ & $7(17.1 \%)$ & 0.077 \\
\hline Overall & $27(65.9 \%)$ & $9(22.0 \%)$ & $<0.01^{*}$ \\
\hline \multicolumn{4}{|c|}{ Transfusion response, $\mathbf{N}(\%)$} \\
\hline $\mathrm{RBC}$ & $6 / 16(37.5 \%)$ & $5 / 17(29.4 \%)$ & 0.721 \\
\hline Platelets & $7 / 14(50.0 \%)$ & $1 / 6(6.7 \%)$ & 0.325 \\
\hline Overall & $11 / 25(44.0 \%)$ & $5 / 18(27.8 \%)$ & 0.278 \\
\hline
\end{tabular}

In subgroup analysis, factors including patients of older age ( $>60$ years), presence of 2 or more cytopenias, good cytogenetic risk, transfusion independence, gene mutation, intermediate-1 risk disease by IPSS, and category 1 or 2 by LR-PSS were associated with a higher OR rate in the decitabine group when compared with that in the BSC group (Figure 2). Also, patients who received decitabine had a significantly higher OR rate regardless of the percentages of the bone marrow blasts.

\section{Patient survival}

The median follow-up for the entire cohort was 40.6 months (IQR: 22.6-50.4 months), with a total of 5 patients lost to follow-up (2 in the decitabine group and 3 in the BSC group). The EFS in the decitabine group (20.6 months) was longer than that in the BSC group (14.3 months), although this was not statistically significant $(\mathrm{p}=0.665)$ (Figure $3 \mathrm{~A})$. In the univariate analyses of factors associated with EFS, patients at $\leq 60$ years of age $(p=0.014)$, with $<5 \%$ bone marrow blasts $(p=0.004)$, achievement of OR $(p$ $=0.005)$, and wild-type DNMT3A $(p=0.013)$ affected the survival favorably. In the multivariate analysis, patients with $<5 \%$ bone marrow blasts $(p=0.008)$ and achievement of OR $(p=0.002)$ remained significantly associated with longer EFS.

The median OS were 28.3 and 36.9 months in the decitabine and BSC groups respectively $(p=0.630)$ (Figure 3B). In the univariate analysis of factors associated with OS, patients at $\leq 60$ years of age ( $p$ $<0.01)$, with $<5 \%$ bone marrow blasts $(\mathrm{p}=0.017)$, transfusion independence $(\mathrm{p}=0.011)$, wild-type DNMT3A ( $p<0.01)$, and wild-type IDH2 ( $p=0.033)$ affected the survival favorably. In the multivariate analysis, patients at $\leq 60$ years of age $(p=0.008),<5 \%$ bone marrow blasts $(p=0.024)$, and transfusion independence $(p<0.01)$ remained significantly associated with longer OS.

\section{Toxicities}

Adverse events were observed frequently in the patients treated with decitabine. In this group, grade $3 / 4$ neutropenia was the most common (41.5\%, $17 / 41)$, and $46.3 \%(19 / 41)$ of patients developed any grade of infection or neutropenic fever. One patient treated with decitabine died of acute cerebral infarction at week- 6 of treatment.

\section{Discussion}

In this study, we found that lower-dose decitabine was clinically effective (OR rate: 65.9\%, $27 / 41$ ), and patients who were transfusion independent before treatment had a higher OR rate. Our findings were consistent with a previous study by Jabbour et al. at a lower-dose decitabine regimen (20 mg/m²/day for 3 days) [14]. Besides, Zeidan AM et al. [21] revealed that the number of transfusions in the 8 weeks before the initiation of hypomethylating agents affected the odds of achieving transfusion independence, and that patients who required more blood transfusion were associated with lower odds of transfusion independence after treatment. Also, our 
survival analysis suggested that decitabine treatment prolonged the EFS for another half a year when compared with BSC treatment, and a longer EFS was associated with the achievement of an OR. In a retrospective analysis by Jabbour et al. [22] concerning the treatment outcome of hypomethylating agents in 438 patients with lower-risk MDS, 77\% (223/290) remained in the lower-risk disease category with the median transformation-free survival of 15 months despite treatment failure. This suggests that hypomethylating agents can delay the progression of the disease. Furthermore, a longer survival has been associated with the achievement of HI [9], especially in patients with intermediate or high-risk features.

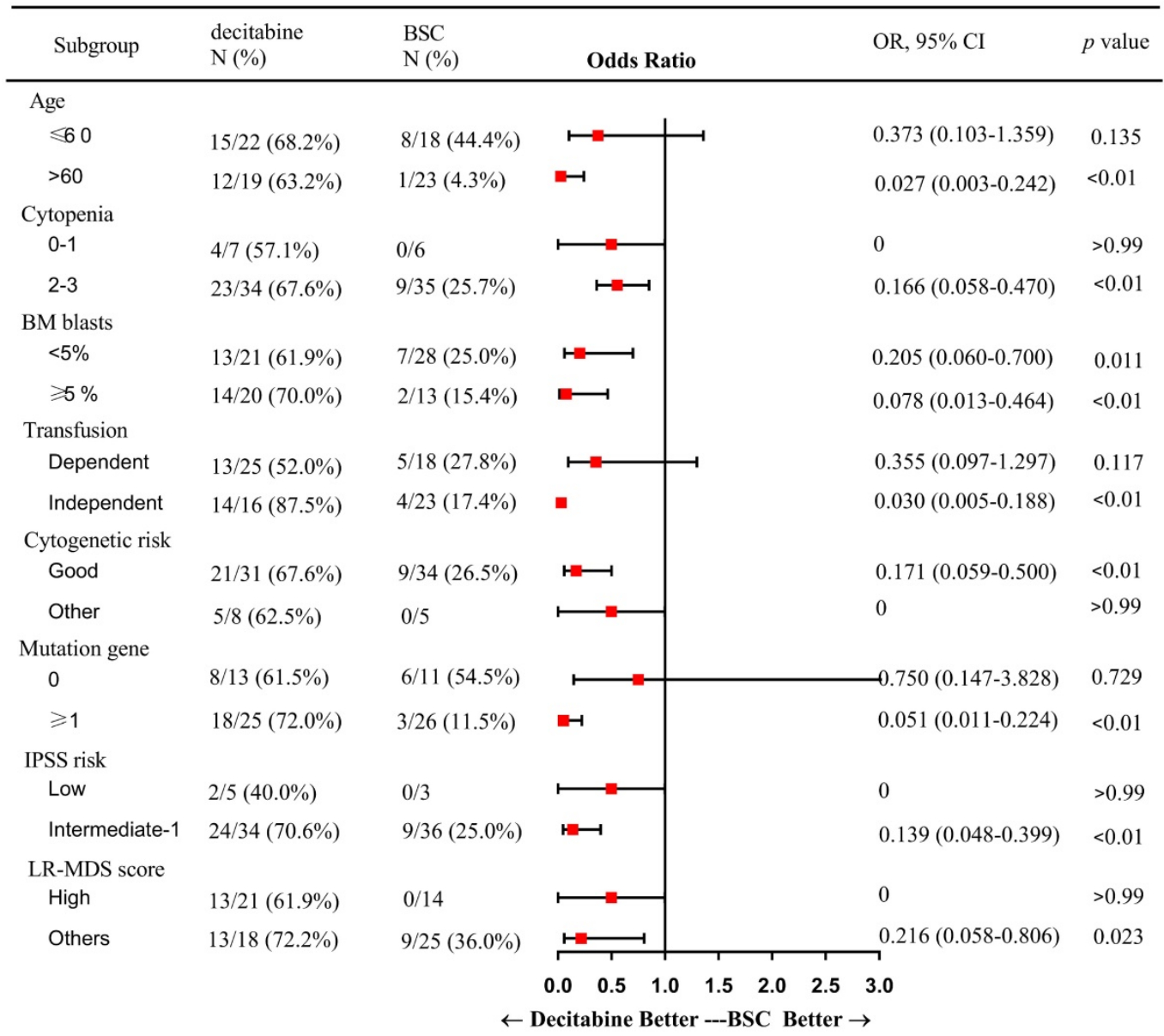

Figure 2. Subgroup analysis: a forest plot showing the odds ratios for overall response of various subgroups by treatment group. Abbreviations: BSC: best supportive care; OR: Odds Ratio; Cl: confidence interval; BM: bone marrow; IPSS: International prognostic scoring system; LR: lower-risk.
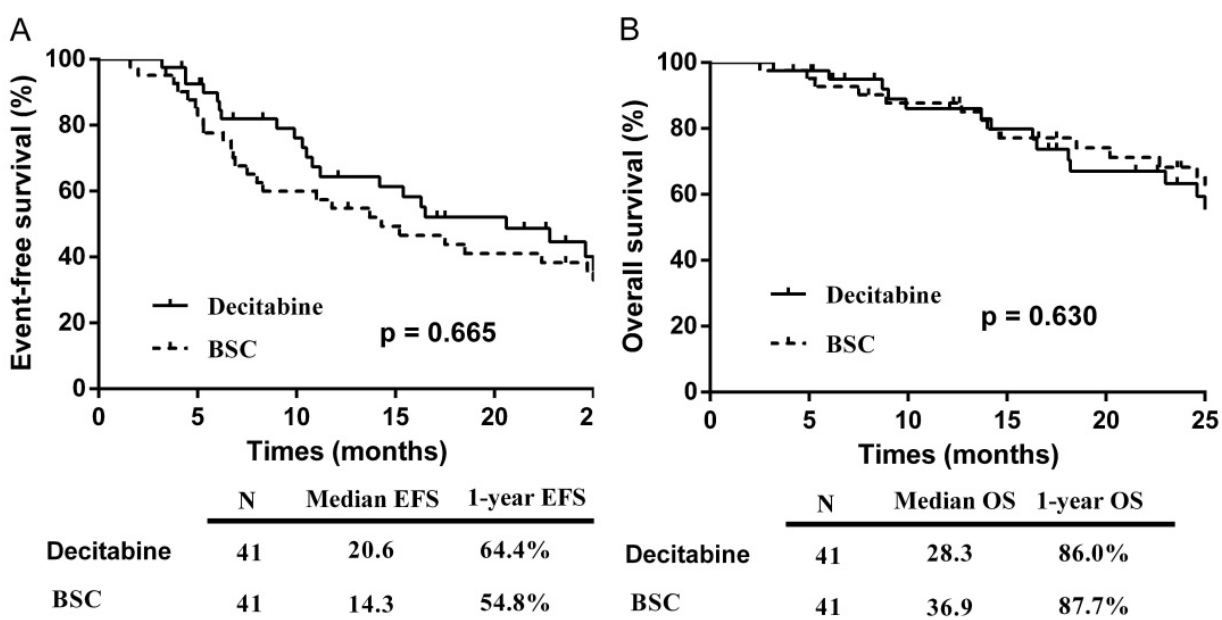

Figure 3. Kaplan-Meier curve: (A) EFS by treatment group; (B) OS by treatment group. Abbreviations: BSC: best supportive care; EFS: event-free survival; OS: overall survival. 
In MDS, more than 40 recurrently mutated genes have been identified. Studies have shown that the TET2 mutation favorably influenced the treatment response to hypomethylating agents [23, 24], while this phenomenon was not observed in the study by Idossa et al. [25]. In our study, the incidence of TET2 mutation was the most common, but mutated TET2 was not associated with a higher OR rate when compared with the wild-type TET2. Nevertheless, patients in the decitabine group with at least 1 gene mutation were observed to have a higher OR rate $(72.0 \%)$. Therefore, patients with the mutated gene may benefit from early intervention with a hypomethylating agent. On the other hand, patients with mutated DNMT3A or IDH2 had been shown to have worse overall survival and more rapid progression to AML, compared to patients with wild-type DNMT3A or IDH2 [7, 26-28]. Our findings also revealed an association of DNMT3A or IDH2 mutation with a shortened EFS or OS, which however were not independent prognostic factors, possibly due to the small sample size of patients harboring each of these mutations.

Several studies have shown that the incidence of adverse events is dependent on the dosage of decitabine. In the study by Lubbert $\mathrm{M}$ et al. [29], a high proportion of patients with intermediate- or high-risk MDS and treated with decitabine (45 $\mathrm{mg} / \mathrm{m}^{2} /$ day for consecutive 3 days) developed Grade 3 to 4 febrile neutropenia (25\%) and infections (57\%). Moreover, in lower-risk patients treated with decitabine $20 \mathrm{mg} / \mathrm{m}^{2} /$ day for consecutive 3 days, Garcia-Manero $G$ et al. [15] revealed a high rate of Grade 3 to 4 adverse events (40\%) and neutropenia $(28 \%, 12 / 43)$. However, when the treatment dose was reduced, the incidence of adverse events was also minimized, as demonstrated by Jabbour E et al. [14] that Grade 3 or more infection or neutropenia were rare when low-dose hypomethylating agents were administered. In our study, the most common toxicities related to decitabine were infection and neutropenia, in which $41.5 \%(17 / 41)$ developed grade 3 to 4 neutropenia and $46.3 \%$ (19/41) suffered any grades of infection. Within 3 months of treatment, one patient $(4.2 \%)$ died of acute cerebral infarction at week-6. Taken into consideration of findings from our studies, our results suggested that the toxicity profiles of decitabine $12 \mathrm{mg} / \mathrm{m}^{2} /$ day (for consecutive 5 days) and $20 \mathrm{mg} / \mathrm{m}^{2} /$ day decitabine (for consecutive 3 days) were comparable in lower-risk MDS patients.

In conclusion, lower-dose decitabine is effective and tolerable in patients with lower-risk MDS. Patients with gene mutations may achieve a higher response rate to decitabine than best supportive care. Further research into lower-risk MDS patients with high-risk gene mutations will shed light on how those mutations may influence the clinical response to decitabine and will provide opportunities for targeted intervention.

\section{Abbreviations}

BSC: best supportive care; IQR: Inter-Quartile Range; BM: bone marrow; WHO: World Health Organization; RAEB: refractory anemia with excess blasts; IPSS: International prognostic scoring system; IPSS-R: IPSS-Revised; LR-PSS: lower-risk PSS; OR: overall response; CR: complete remission; PR: partial remission; $\mathrm{mCR}$ : marrow $\mathrm{CR}$; $\mathrm{HI}$ : hematologic improvement; RBC: red blood cell; HSCT: hematopoietic stem cell transplantation; OR: Odds Ratio (Figure 2); CI: confidence interval; EFS: event-free survival; OS: overall survival.

\section{Acknowledgements}

This work was supported by The National Natural Science Foundation of China grants (81470290).

\section{Trial registration}

Chinese Clinical Trial Registry. CHiCTR-IPR15006454 (September 2013).

\section{Authorship}

H.T. and L.Y. designed the study. L.Y. and H.T. wrote the manuscript. Y.L. analyzed and arranged the data. L.Y., C.M. and Y.R. performed the gene mutation analysis. X.Z., L.M., W.X., J.W., H.J., L.Z. and H.Z. provided patient samples and data. H.T. guided the project design and article modification. We sincerely thank the sample donors and clinical investigators who participated in this study.

\section{Competing Interests}

The authors have declared that no competing interest exists.

\section{References}

1. Gangat N, Patnaik MM, Tefferi A. Myelodysplastic syndromes: Contemporary review and how we treat. American journal of hematology. 2016; 91: 76-89.

2. Vardiman JW. Hematopathological concepts and controversies in the diagnosis and classification of myelodysplastic syndromes. Hematology American Society of Hematology Education Program. 2006: 199-204.

3. Steensma DP. Myelodysplastic Syndromes: Diagnosis and Treatment. Mayo Clinic proceedings. 2015; 90: 969-83.

4. Garcia-Manero G, Shan J, Faderl S, Cortes J, Ravandi F, Borthakur G, et al. A prognostic score for patients with lower risk myelodysplastic syndrome. Leukemia. 2008; 22: 538-43.

5. Bejar R, Stevenson KE, Caughey BA, Abdel-Wahab O, Steensma DP, Galili N, et al. Validation of a prognostic model and the impact of mutations in patients with lower-risk myelodysplastic syndromes. Journal of clinical oncology: official journal of the American Society of Clinical Oncology. 2012; 30: 3376-82.

6. Bejar R, Stevenson K, Abdel-Wahab O, Galili N, Nilsson B, Garcia-Manero G, et al. Clinical effect of point mutations in myelodysplastic syndromes. The New England journal of medicine. 2011; 364: 2496-506.

7. Jiang L, Luo Y, Zhu S, Wang L, Ma L, Zhang H, et al. Mutation status and burden can improve prognostic prediction of patients with lower-risk myelodysplastic syndromes. Cancer science. 2020; 111: 580-91. 
8. Bejar R. Implications of molecular genetic diversity in myelodysplastic syndromes. Current opinion in hematology. 2017; 24: 73-8.

9. Lee JH, Kim YJ, Sohn SK, Yoon SS, Kim H, Cheong JW, et al. Benefits of hypomethylating therapy in IPSS lower-risk myelodysplastic syndrome patients: A retrospective multicenter case series study. Leukemia research. 2017; 60: 135-44.

10. Lee JH, Jang JH, Park J, Park S, Joo YD, Kim YK, et al. A prospective multicenter observational study of decitabine treatment in Korean patients with myelodysplastic syndrome. Haematologica. 2011; 96: 1441-7.

11. Li X, Song Q, Chen Y, Chang C, Wu D, Wu L, et al. Decitabine of reduced dosage in Chinese patients with myelodysplastic syndrome: a retrospective analysis. PloS one. 2014; 9: e95473.

12. Yang AS, Doshi KD, Choi SW, Mason JB, Mannari RK, Gharybian V, et al. DNA methylation changes after 5-aza-2'-deoxycytidine therapy in patients with leukemia. Cancer research. 2006; 66: 5495-503.

13. Issa JP, Garcia-Manero G, Giles FJ, Mannari R, Thomas D, Faderl S, et al. Phase 1 study of low-dose prolonged exposure schedules of the hypomethylating agent 5-aza-2'-deoxycytidine (decitabine) in hematopoietic malignancies. Blood. 2004; 103: 1635-40.

14. Jabbour E, Short NJ, Montalban-Bravo G, Huang X, Bueso-Ramos C, Qiao W, et al. Randomized phase 2 study of low-dose decitabine vs low-dose azacitidine in lower-risk MDS and MDS/MPN. Blood. 2017; 130: 1514-22.

15. Garcia-Manero G, Jabbour E, Borthakur G, Faderl S, Estrov Z, Yang H, et al. Randomized open-label phase II study of decitabine in patients with low- or intermediate-risk myelodysplastic syndromes. Journal of clinical oncology: official journal of the American Society of Clinical Oncology. 2013; 31: 2548-53.

16. Vardiman JW, Thiele J, Arber DA, Brunning RD, Borowitz MJ, Porwit A, et al. The 2008 revision of the World Health Organization (WHO) classification of myeloid neoplasms and acute leukemia: rationale and important changes. Blood. 2009; 114: 937-51.

17. Cheson BD, Greenberg PL, Bennett JM, Lowenberg B, Wijermans PW, Nimer $\mathrm{SD}$, et al. Clinical application and proposal for modification of the International Working Group (IWG) response criteria in myelodysplasia. Blood. 2006; 108: 419-25.

18. Trotti A, Colevas AD, Setser A, Rusch V, Jaques D, Budach V, et al. CTCAE v3.0: development of a comprehensive grading system for the adverse effects of cancer treatment. Seminars in radiation oncology. 2003; 13: 176-81.

19. Bacher U, Kohlmann A, Haferlach T. Mutational profiling in patients with MDS: ready for every-day use in the clinic? Best practice \& research Clinical haematology. 2015; 28: 32-42.

20. Kohlmann A, Grossmann V, Nadarajah N, Haferlach T. Next-generation sequencing - feasibility and practicality in haematology. British journal of haematology. 2013; 160: 736-53.

21. Zeidan AM, Zhu W, Stahl M, Wang R, Huntington SF, Giri S, et al. RBC transfusion independence among lower risk MDS patients receiving hypomethylating agents: a population-level analysis. Leukemia \& lymphoma. 2019: 1-7.

22. Jabbour EJ, Garcia-Manero G, Strati P, Mishra A, Al Ali NH, Padron E, et al. Outcome of patients with low-risk and intermediate-1-risk myelodysplastic syndrome after hypomethylating agent failure: a report on behalf of the MDS Clinical Research Consortium. Cancer. 2015; 121: 876-82.

23. Itzykson $\mathrm{R}$, Kosmider $\mathrm{O}$, Cluzeau $\mathrm{T}$, Mansat-De Mas V, Dreyfus $\mathrm{F}$, Beyne-Rauzy $\mathrm{O}$, et al. Impact of TET2 mutations on response rate to azacitidine in myelodysplastic syndromes and low blast count acute myeloid leukemias. Leukemia. 2011; 25: 1147-52.

24. Bejar R, Lord A, Stevenson K, Bar-Natan M, Perez-Ladaga A, Zaneveld J, et al. TET2 mutations predict response to hypomethylating agents in myelodysplastic syndrome patients. Blood. 2014; 124: 2705-12.

25. Idossa D, Lasho TL, Finke CM, Ketterling RP, Patnaik MM, Pardanani A, et al. Mutations and karyotype predict treatment response in myelodysplastic syndromes. American journal of hematology. 2018; 93: 1420-6.

26. Walter MJ, Ding L, Shen D, Shao J, Grillot M, McLellan M, et al. Recurrent DNMT3A mutations in patients with myelodysplastic syndromes. Leukemia. 2011; 25: 1153-8.

27. Jin J, Hu C, Yu M, Chen F, Ye L, Yin X, et al. Prognostic value of isocitrate dehydrogenase mutations in myelodysplastic syndromes: a retrospective cohort study and meta-analysis. PloS one. 2014; 9: e100206.

28. Lin $\mathrm{P}$, Luo Y, Zhu S, Maggio D, Yang H, Hu C, et al. Isocitrate dehydrogenase 2 mutations correlate with leukemic transformation and are predicted by 2-hydroxyglutarate in myelodysplastic syndromes. Journal of cancer research and clinical oncology. 2018; 144: 1037-47.

29. Lubbert M, Suciu S, Baila L, Ruter BH, Platzbecker U, Giagounidis A, et al. Low-dose decitabine versus best supportive care in elderly patients with intermediate- or high-risk myelodysplastic syndrome (MDS) ineligible for intensive chemotherapy: final results of the randomized phase III study of the European Organisation for Research and Treatment of Cancer Leukemia Group and the German MDS Study Group. Journal of clinical oncology: official journal of the American Society of Clinical Oncology. 2011; 29: 1987-96. 